\title{
The 5th International Workshop on Social Web for Disaster Management (SWDM'18)
}

\author{
Collective Sensing, Trust, and Resilience in Global Crises
}

\author{
Yu-Ru Lin \\ School of Computing and \\ Information, University of \\ Pittsburgh, PA, USA \\ yurulin@pitt.edu
}

\author{
Carlos Castillo \\ Universitat Pompeu Fabra \\ Barcelona, Catalonia, Spain \\ chato@acm.org
}

\author{
Jie Yin \\ CSIRO \\ Epping, NSW, Australia \\ Jie.Yin@data61.csiro.au
}

\begin{abstract}
During large-scale emergencies such as natural and man-made disasters, a massive amount of information is posted by the public in social media. Collecting, aggregating, and presenting this information to stakeholders can be extremely challenging, particularly if an understanding of the "big picture" is sought. This international workshop, the fifth in the series, is a key venue for researchers and practitioners to discuss research challenges and technical issues around the usage of social media in disaster management.

Workshop's website: https://sites.google.com/site/swdm2018/
\end{abstract}

\section{CCS CONCEPTS}

- Information systems $\rightarrow$ Web mining; • Human-centered computing $\rightarrow$ Social networking sites;

\section{KEYWORDS}

social media, emergency management, disaster response

\section{ACM Reference Format:}

Yu-Ru Lin, Carlos Castillo, and Jie Yin. 2018. The 5th International Workshop on Social Web for Disaster Management (SWDM'18): Collective Sensing, Trust, and Resilience in Global Crises. In WSDM 2018: The Eleventh ACM International Conference on Web Search and Data Mining, February 5-9, 2018, Marina Del Rey, CA, USA. ACM, New York, NY, USA, 2 pages. https: //doi.org/10.1145/3159652.3160594

\section{WORKSHOP OVERVIEW}

The proliferation of social media platforms together with the wide adoption of smartphone devices has transformed how we communicate and share news. During large-scale emergencies, such as natural disasters or armed attacks, victims, responders, and volunteers increasingly use social media to post situation updates and to request and offer help. There are a number of challenges associated with near-real-time processing of vast volumes of information in a way that makes sense for people directly affected, for volunteer organizations, and for official emergency response agencies. As massive amount of relevant data are accumulated, there is a

Permission to make digital or hard copies of part or all of this work for personal or classroom use is granted without fee provided that copies are not made or distributed for profit or commercial advantage and that copies bear this notice and the full citation on the first page. Copyrights for third-party components of this work must be honored.

For all other uses, contact the owner/author(s).

WSDM 2018, February 5-9, 2018, Marina Del Rey, CA, USA

(C) 2018 Copyright held by the owner/author(s)

ACM ISBN 978-1-4503-5581-0/18/02.

https://doi.org/10.1145/3159652.3160594 growing need for developing advanced techniques to harness this large-scale data to gain an understanding of the "big picture" of an emergency, and to detect and predict how a disaster could develop.

The Social Web for Disaster Management (SWDM) international workshop series, now in its fifth edition, is a key venue for computer scientists, government officials, emergency agencies, and other interested participants to discuss technical issues and research challenges in the understanding of digitally-mediated, social and technical systems during mass emergencies. The workshop theme for this year is "Collective Sensing, Trust, and Resilience in Global Crises," through which we aim to facilitate interdisciplinary discussion on a range of emergent challenges facing the world, from harnessing big data on the Web that facilitates disaster and risk management, to engaging citizen participation in disaster response, establishing trust in global and local communities, designing socio-technical systems, and building resilience to disasters through Internet of Things and ubiquitous intelligence.

\section{RELEVANCE AND SIGNIFICANCE}

Over the last decade, nearly 2.5 billion people have been affected by disasters, which claimed 1.3 million lives and costed US \$2 trillion in economic losses. Large-scale emergencies including natural hazards and man-made disasters are an enormous threat to societies. There is an indisputably increasing need for building new data mining tools to strengthen disaster management and humanitarian response, by harnessing the data on the Web and the Social Web. The scope and focus of this workshop is well aligned with the theme of WSDM - which also emphasizes on "the practical and principled novel models of search and data mining on the Web and the Social Web." In particular, disaster-related data are big and noisy, and advanced algorithm design and analysis, and in-depth experimental analysis solutions are needed for a variety of applications in disaster scenarios.

Based on our prior successful experience in running related workshops in conjunction with conferences, this year we seek to deepen the research conversation from the aspects of knowledge management and discovery, and unstructured and spatiotemporal data mining, through engaging the researchers and practitioners from the WSDM community. Modern disaster management involves analysis of massive, noisy, and heterogeneous data sources, including urban sensors and various types of social media, which change very rapidly throughout a crisis. We would like to bring together WSDM participants who are interested in research challenges in crisis domains, such as stream processing of microtext and images, 
situational information mining, dynamic network analysis, data quality assurance, and knowledge discovery applied to large-scale crisis data.

\section{TARGET AUDIENCE}

The target audience includes academic and industry researchers as well as government agencies. We welcome interested participants to discuss issues and challenges in the understanding of large-scale social media data during mass emergencies, especially researchers and practitioners who are interested in the area of disaster related data mining and machine learning approaches.

\section{WORKSHOP FORMAT}

The workshop will include four elements: keynotes, talks, demonstrations and panel discussion. As in previous editions, each session will be allocated with sufficient time for questions, interaction, and discussions. We plan to include two keynotes.

\section{Keynote speaker: Dr. Carter T. Butts}

"Attention, Amplification, Resilience, and Hazard Messaging at the Organizational/Public Interface"

Carter T. Butts is a Professor in the Departments of Sociology, Statistics, and Electrical Engineering and Computer Science at the University of California Irvine, where he directs the Center for Networks and Relational Analysis in the California Institute for Telecommunications and Information Technology. Professor Butts received his doctoral degree from Carnegie Mellon University, and his bachelor's degree from Duke University; he holds early career awards from the American Sociological Association and the International Network for Social Network Analysis, and is currently chair-elect of the ASA Section on Mathematical Sociology. Professor Butts's research involves the development and application of mathematical, computational, and statistical techniques to theoretical and methodological problems within the areas of social and biological network analysis, mathematical sociology, quantitative methodology, and human judgment and decision making. He has published extensively on communication in hazard response contexts, including work on emergent coordination in the World Trade Center Disaster, organizational collaboration in the aftermath of Hurricane Katrina, message passing following the Boston Marathon Bombing, and attentional dynamics during wildfires.

\section{Keynote speaker: Dr. Paige Maas}

\section{"Leveraging Facebook Data for Disaster Response"}

Paige Maas is a Data Scientist on the Core Data Science team at Facebook, where she has worked on understanding user experience and interactions and helping build tools to make that experience better. She earner her $\mathrm{PhD}$ in Biostatistics from Johns Hopkins University and did her dissertation research at the US National Cancer Institute, where she developed methods for synthesizing data sources to build risk prediction models for breast cancer. At Facebook, her current focus is on building data products that can be used by humanitarian organizations in disaster response.

\section{WEBSITE}

The workshop has a companion website where talk slides will be posted: https://sites.google.com/site/swdm2018/

\section{ORGANIZERS}

$Y u-R u$ Lin. (yurulin@pitt.edu) is an assistant professor at the School of Computing and Information, University of Pittsburgh. Her research interests include human mobility, social and political network dynamics, and computational social science. She has developed computational approaches for mining and visualizing large-scale, time-varying, heterogeneous, multi-relational, and semi structured data. Her current research focuses on extracting systemlevel features from big data sets, including social media data, for studying social dynamics, particularly under exogenous events such as emergencies. Her work has appeared in prestigious scientific venues including WWW, SIGKDD, InfoVis, ACM TKDD, ACM TOMCCAP, IEEEP and PLOS ONE. She has served in program committees for international data and web mining conferences and has organized several workshops in data science including 2014 KDD Workshop on Learning about Emergencies from Social Information (KDD-LESI). She co-organized the SWDM 2016.

Carlos Castillo. (chato@acm.org) is a distinguished research professor at Universitat Pompeu Fabra. Carlos is an active researcher on web mining and search, with more than 75 papers in top-tier international conferences and journals, including a book on Big Crisis Data, a book on Information and Influence Propagation, and a monograph on Adversarial Web Search. Carlos received his Ph.D from the University of Chile, and was senior scientist at Yahoo! Research, principal scientist at the Qatar Computing Research Institute, and director of research for data science at Eurecat. He has served in the PC or SPC of all major conferences in his area (WWW, WSDM, KDD, SIGIR, CIKM) and is part of the editorial committee of ACM Transactions on the Web and Internet Research. He was Program Committee Co-Chair of ACM Digital Health 2016, and was Program Committee Co-chair of WSDM 2014, co-organized the Adversarial Information Retrieval Workshop and Web Spam Challenge in 2007 and 2008, the ECML/PKDD Discovery Challenge in 2010 and 2014, the Web Quality Workshop from 2011 to 2014, and the Social Web for Disaster Management Workshop in 2015 and 2016.

fie Yin. (jie.yin@csiro.au) is a senior research scientist at CSIRO, Australia. Her main research interests include data mining, machine learning, and their applications to text mining, social media mining and pervasive computing. She received the Ph.D. degree in Computer Science from Hong Kong University of Science and Technology. Her research experience includes activity recognition from sensor data, text mining from social media and Web forums, active learning and transfer learning in social networks. Jie is an active researcher in data mining with more than 40 research papers published in top-tier international conferences (AAAI, IJCAI, CIKM, ICDM, etc.) and journals (TKDE, TPAMI, TOIS, TOSN, etc.). She has served as the PC of all major conferences in her area including ICDM, KDD, AAAI, CIKM, etc. She was co-organizer of the 3rd International Workshop on Social Web for Disaster Management (SWDM 2015). 